\title{
Lignes directrices provisoires pour les analyses effectuées en laboratoire visant la détection des infections à Mycobacterium non tuberculeuses (MNT) chez les patients en phase post-opératoire exposés à des échangeurs thermiques
}

\author{
$K$ Antonation (coprésidence à l'échelle fédérale) ${ }^{1}, S$ Patel (coprésidence à l'échelle provinciale)2 \\ J Trumble Waddell', P Guillaume Poliquin ${ }^{1}$, DC Alexander ${ }^{3}$, L Hoang ${ }^{4}$, D Farrell ${ }^{5}$, R Garceau 6 , \\ D Haldane ${ }^{7}$, F Jamieson ${ }^{2}$, R Marchand ${ }^{8}$, A MacKeen 9 , D Marcino ${ }^{9 \star}$, S Theriault ${ }^{1}$, GJ Tyrrell10, \\ G Zahariadis ${ }^{11}, \mathrm{~N}$ Zelyas ${ }^{10}$ au nom du Réseau des laboratoires de santé publique du Canada
}

\section{Résumé}

Les conseils contenus dans le présent document devraient être lus conjointement avec les lois, les politiques et les règlements provinciaux, territoriaux et locaux pertinents. Les mesures recommandées ne devraient pas être considérées comme des normes rigides, mais plutôt comme des principes et des recommandations visant à guider l'élaboration de lignes directrices.

Les présents avis sont fondés sur les données scientifiques actuellement disponibles et reposent sur un principe de précaution lorsque les preuves sont insuffisantes ou non concluantes. Elles ont été approuvées aux fins de publication le 5 décembre 2016. Elles sont sujettes à examen et à changement à mesure que de nouveaux renseignements seront disponibles.

Les principaux changements apportés à la présente version comprennent des ajouts à ce qui suit : nombre de cas signalés à ce jour, maladies similaires à la sarcoïdose comme indicateur, travaux portant sur le séquençage du génome, liens vers les signalements des services de laboratoire provinciaux et territoriaux et de Santé Canada.
Affiliations

${ }^{1}$ Laboratoire national de microbiologie, Winnipeg (Manitoba)

2 Santé publique Ontario, Toronto (Ontario)

${ }^{3}$ Laboratoire provincial de Cadham, Winnipeg (Manitoba)

${ }^{4}$ Centre de contrôle des maladies de la Colombie-Britannique, Vancouver (Colombie-Britannique) ${ }^{5}$ Laboratoire de lutte contre les maladies de la Saskatchewan, Regina (Saskatchewan)

${ }^{6}$ Hôpital Dr-George-L.-Dumont, Moncton (Nouveau-Brunswick)

${ }^{7}$ Centre des sciences de la santé Queen Elisabeth II, Halifax (Nouvelle-Écosse)

${ }^{8}$ Laboratoire de santé publique du Québec,

Sainte-Anne-de-Bellevue (Québec)

9 Réseau des laboratoires de santé publique du Canada Winnipeg (Manitoba)

${ }^{10}$ Laboratoire provincial de santé publique de l'Alberta, Edmonton (Alberta)

${ }^{11}$ Laboratoire provincial de santé publique, Services de microbiologie Eastern Health. St. John's (Terre-Neuve)

*Correspondance : dionne. marcino@phac.aspc.gc.ca

Citation proposée : Antonation K (coprésidence à l'échelle fédérale), Patel S (coprésidence à l'échelle provinciale), Trumble Waddell J, Guillaume Poliquin P, Alexander DC, Hoang L et al. Lignes directrices provisoires pour les analyses effectuées en laboratoire visant la détection des infections à Mycobacterium non tuberculeuses (MNT) chez les patients en phase post-opératoire exposés à des échangeurs thermiques. Relevé des maladies transmissibles au Canada. 2017;43(1):27-31. https://doi.org/10.14745/ccdr.v43i01a05f

\section{Portée}

Le présent document décrit les critères applicables aux essais de laboratoire et les échantillons à prélever chez les sujets symptomatiques ayant été exposés à un échangeur thermique pendant une intervention chirurgicale à cœur ouvert réalisée depuis le $1^{\text {er }}$ novembre 2011.

\section{Contexte}

Une éclosion récente de cas d'infection à Mycobacterium chimaera a été détectée à l'échelle mondiale chez des patients ayant subi une intervention chirurgicale à cœur ouvert en présence d'échangeurs thermiques contaminés. À ce jour, 52 cas ont été répertoriés en Europe et 2 au Canada (11).

Il y a de nombreuses incertitudes en ce qui a trait : 1) à l'ampleur du risque d'infection et aux facteurs influant sur le 
risque d'infection, 2) au tableau clinique de la maladie et 3) à la meilleure façon de gérer les appareils.

À l'heure actuelle, le risque pour les patients est jugé faible. Des estimations du risque seront fournies au fur et à mesure que l'on obtiendra d'autres renseignements sur la situation.

Le Réseau des laboratoires de santé publique du Canada et ses partenaires souhaitent aider les laboratoires en leur fournissant ces recommandations provisoires.

Le présent document portera sur les points suivants : 1 ) définition des patients à risque, pour établir les critères applicables aux essais de laboratoire, 2) recommandations liées au prélèvement d'échantillons et au dépistage d'une infection à M. chimaera chez les patients.

\section{Présentations cliniques* associées à l'infection à Mycobacterium non tuberculeuse post-opératoire}

Dans la majorité des cas, les symptômes apparaissent de 3 mois à 5 ans (médiane : 18 mois) après la chirurgie; les symptômes sont notamment de la fièvre, de la fatigue, un essoufflement, des sueurs nocturnes, des douleurs articulaires ou musculaires et une perte de poids inexpliquée $(1,3,7)$. Les manifestations cardiaques sont notamment une endocardite sur prothèse valvulaire, une infection sur prothèse vasculaire, un abcès paravalvulaire, ainsi qu'un pseudo anévrisme ou un anévrisme mycotique $(7,10)$. Les manifestations extracardiaques sont notamment une infection osseuse (ostéomyélite, spondylodiscite), une infection de la plaie de sternotomie, une médiastinite, une hépatite et une bactériémie $(3,7,10)$. Des manifestations oculaires dues à des emboles (panuvéite, choroïdite multifocale, choriorétinite) sont observées chez environ $50 \%$ des patients (3). Les manifestations immunologiques englobent notamment les suivantes : arthrite, vascularite cérébrale, pneumonite, myocardite, néphrite granulomateuse $(7,10)$. Une splénomégalie est observée chez environ $80 \%$ des cas (3), de même qu'une atteinte de la moelle osseuse avec cytopénie. Des recommandations récentes ont soulevé l'attention face aux maladies granulomateuses, particulièrement celles qui s'apparent à la sarcoïdose (11). Des cas de $M$. chimaera chez les patients qui avaient reçu un diagnostic initial de sarcoïdose ont été rapportés.

\section{Critères d'analyse des patients}

\section{Critère 1: Exposition au risque}

Les patients doivent avoir subi une chirurgie cardiothoracique dans le passé. En raison de la période d'incubation prolongée, ce critère est considéré comme étant rempli si les patients ont subi une telle intervention depuis le mois de novembre 2011.

Des articles publiés en Allemagne ( 5 cas) $)^{2}$, en Suisse $(6 \mathrm{cas})^{1}$ et au Royaume-Uni (17 (cas) ${ }^{9}$ montrent que la plupart des patients présentaient une endocardite, un abcès paravalvulaire, une infection au site de l'intervention ou une bactériémie associés à un pontage aortocoronarien, à un remplacement valvulaire ou à une réparation valvulaire. Les signes et symptômes accompagnateurs les plus courants étaient notamment de la fatigue, de la fièvre, une hépatite, une insuffisance rénale, une splénomégalie et une pancyopénie.
Attention : Des cas isolés ont été observés chez des patients ayant subi un autre type d'intervention dans une salle où se trouvait un échangeur thermique en fonctionnement. Bien que I'on ne considère habituellement pas ces patients comme étant à risque, ceux-ci pourraient être soumis à un test de dépistage d'une infection à MNT s'ils présentent un syndrome clinique compatible (voir ci-dessous).

\section{Critère 2 : Syndrome clinique compatible}

Dans l'ensemble, les patients présentent des symptômes non spécifiques, ce qui complique la tâche de distinguer les infections dues à des MNT des infections dues à d'autres organismes, plus courants. À cette fin, un syndrome compatible est jugé présent dans les cas ci-dessous :

- Signes généraux : fièvre récurrente ou prolongée, fatigue, essoufflement, perte de poids, sueurs nocturnes, douleurs articulaires ou musculaires

- Manifestations cardiaques : endocardite sur prothèse valvulaire ou infection sur prothèse vasculaire

- Manifestations extracardiaques : infection osseuse, infection de la plaie de sternotomie, médiastinite, hépatite, bactériémie, infection oculaire (panuvéite, choroïdite multifocale, choriorétinite)

- Manifestations immunologiques/emboliques : splénomégalie, cytopénie

- Nourrissons : épisodes fébriles et retard de croissance

Ces symptômes doivent 1) être apparus après l'intervention chirurgicale ou, 2) s'ils étaient présents avant l'intervention chirurgicale, ils doivent avoir empiré considérablement après I'intervention ET avoir été présents pendant trois semaines ou plus. La persistance de ces symptômes non spécifiques au-delà de la période de trois semaines permet d'éliminer les autres infections qui sont généralement diagnostiquées ou qui se résolvent habituellement au cours d'une telle période. En l'absence d'un diagnostic (de maladie infectieuse ou non infectieuse), les patients présentant des symptômes inexpliqués devraient être soumis à un test de dépistage d'une infection à M. chimaera.

\section{Facteurs importants à prendre en considération pour ces analyses}

- À la lumière des données dont on dispose actuellement, les sujets asymptomatiques qui ont subi une intervention chirurgicale à cœur ouvert ne devraient pas subir de test de dépistage d'une infection à $M$. chimaera.

- Il peut être déraisonnable d'attendre trois semaines ou plus si les symptômes du patient sont graves ou s'il serait trop compliqué d'effectuer un suivi parce que la santé du patient est trop fragile ou que l'accès est difficile pour des raisons géographiques. Dans de telles circonstances exceptionnelles, il est possible de faire subir un test de dépistage au patient sans attendre.

\section{Échantillons}

Les échantillons suivants doivent être prélevés en vue d'une culture mycobactérienne chez les patients admissibles, en 
fonction des recommandations concernant les essais de laboratoire :

Les échantillons cliniques prélevés de sites stériles (tableau 1), par exemple du sang, un écoulement purulent, des tissus frais, etc., doivent être envoyés en vue d'une culture mycobactérienne et d'une recherche de bacilles acido-résistants, avec la demande d'analyse (Annexe 1 : Liens vers les services de laboratoire provinciaux). Il est à noter que $M$. chimaera a une croissance lente et que la détection de cette bactérie par culture peut nécessiter une incubation de 6 à 8 semaines. II est possible que M. chimaera ne soit pas détecté au cours des premiers stades de I'infection.

\section{Tableau 1 : Analyses cliniques visant la détection des cas potentiels d'infection aux mycobactéries non tuberculeuse à la suite d'une chirurgie cardiaque}

\begin{tabular}{|c|c|}
\hline $\begin{array}{c}\text { Symptômes cliniques/ } \\
\text { exposition }\end{array}$ & $\begin{array}{c}\text { Recommandation en } \\
\text { matière d'analyse et } \\
\text { d'échantillon }\end{array}$ \\
\hline $\begin{array}{l}\text { Asymptomatique } \\
\text { ET } \\
\text { Chirurgie cardiothoracique } \\
\text { APRĖS le } 1^{\text {er }} \text { novembre } 2011\end{array}$ & Aucune \\
\hline $\begin{array}{l}\text { Symptomatique }{ }^{1} \\
\text { - Signes généraux : fièvre } \\
\text { récurrente ou prolongée, } \\
\text { fatigue, essoufflement, perte } \\
\text { de poids, sueurs nocturnes } \\
\text { - Manifestations cardiaques : } \\
\text { endocardite sur prothèse } \\
\text { valvulaire ou infection sur } \\
\text { prothèse vasculaire } \\
\text { Manifestations } \\
\text { extracardiaques : infection } \\
\text { osseuse, infection de la plaie } \\
\text { de sternotomie, médiastinite, } \\
\text { hépatite, bactériémie, } \\
\text { infection oculaire } \\
\text { (panuvéite, choroïdite } \\
\text { multifocale, choriorétinite) } \\
\text { Manifestations } \\
\text { immunologiques/ } \\
\text { emboliques : splénomégalie, } \\
\text { cytopénie } \\
\text { Nourrissons : épisodes } \\
\text { fébriles et retard de } \\
\text { croissance } \\
\text { ET } \\
\text { Chirurgie à cœur ouvert } \\
\text { de } 3 \text { mois à } 5 \text { ans avant } \\
\text { l'apparition des symptômes }\end{array}$ & 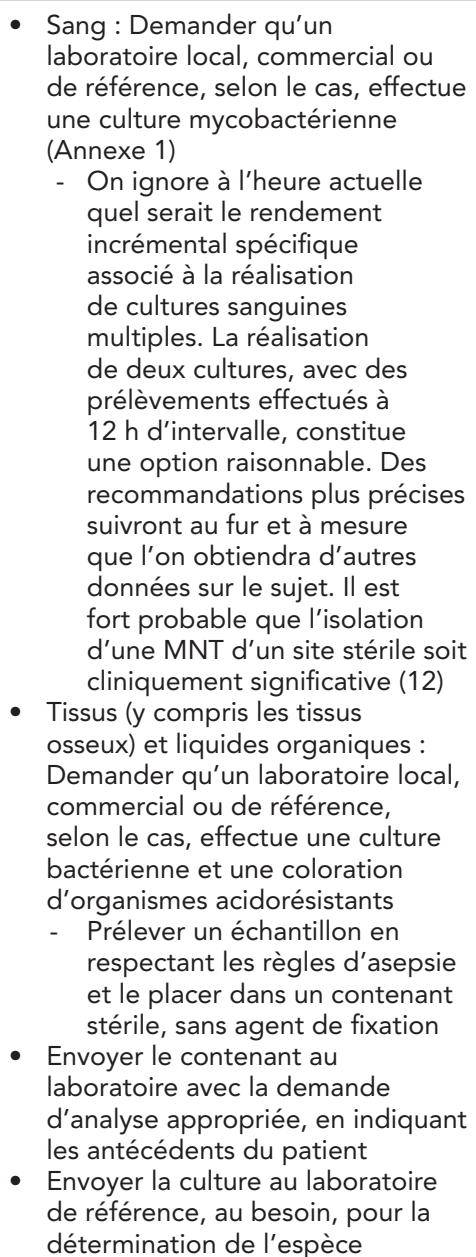 \\
\hline
\end{tabular}

'Définition de symptomatique : Une enquête menée sur une infection aux mycobactéries non tuberculeuses chez les patients atteints d'une maladie prolongée (3 semaines ou plus) ET lorsqu'un autre diagnostic n'est pas disponible par un examen d'usage pour éliminer les agents étiologiques courants
Les cultures positives identifiées comme étant associées à un complexe $M$. avium-intracellulare doivent être envoyées à un laboratoire de référence pour séquençage du gène codant I'ARNr 16S (ou des gènes hsp65/ITS), pour confirmer qu'il s'agit bien de l'espèce Mycobacterium chimaera (https://cnphi.canada. $\mathrm{ca} / \mathrm{gts} /$ reference-diagnostic-test/5054?labld=1004). Pour envoyer les isolats au laboratoire de référence, il est recommandé d'utiliser une culture pure, avec un milieu solide ou liquide (minimum de $4 \mathrm{ml}$ ).

Les isolats potentiellement reliés à cette éclosion sont présentement soumis à un séquençage du génome dans le cadre d'un effort national de collaboration. Les résultats sont à venir.

\section{Vérification des échangeurs thermiques et du milieu environnant}

Santé Canada (http://healthycanadians.gc.ca/ recall-alert-rappel-avis/hc-sc/2016/60662a-fra. php\#issue-problem) peut donner des conseils sur la mise à l'essai des échangeurs thermiques.

\section{Déclaration des effets indésirables associés aux dispositifs médicaux}

Santé Canada encourage les professionnels de la santé à signaler tout cas d'infection qui semble être associé à l'utilisation d'un dispositif médical. Le formulaire Rapport d'incident et les directives associées aux appareils médicaux (http://www.hc-sc. gc.ca/dhp-mps/compli-conform/info-prod/md-im/index-fra. $\mathrm{php} / \mathrm{h}$ ) peuvent être consultés sur le site Web Santé Canada.

\section{Remerciements}

Les auteurs désirent remercier les membres du groupe de travail sur la prévention et le contrôle des infections de l'Agence de la santé publique du Canada pour leurs conseils et leur contribution dans l'élaboration de ces lignes directrices provisoires.

De plus, les auteurs désirent remercier Kathleen Dunn de l'Agence de la santé publique du Canada pour sa contribution dans ce travail.

\section{Conflits d'intérêts}

Aucun.

\section{Financement}

Le soutien administratif pour ce travail a été assuré par l'Agence de la santé publique du Canada. 


\section{Références}

1. Sax, Hugo, et al. "Prolonged outbreak of Mycobacterium chimaera infection after open-chest heart surgery." Clinical Infectious Diseases 61(1) (2015): 67-75.

2. Haller, Sebastian, et al. "Contamination during production of heater-cooler units by Mycobacterium chimaera potentia cause for invasive cardiovascular infections: results of an outbreak investigation in Germany, April 2015 to February 2016." Euro Surveillance 21(17) (2016).

3. Tan, Nicholas, et al. "Disseminated Mycobacterium chimaera Infection After Cardiothoracic Surgery." Open Forum Infectious Diseases. 3(3). (2016).

4. Perkins, Kiran M. "Mycobacterium chimaera Contamination of Heater-Cooler Devices Used in Cardiac Surgery-United States." MMWR. Morbidity and Mortality Weekly Report 65(40) (2016): 1117-1118.

5. Schreiber, Peter W., et al. "Reemergence of Mycobacterium chimaera in Heater-Cooler Units despite Intensified Cleaning and Disinfection Protocol." Emerging Infectious Diseases 22(10) (2016): 1830-1833.

6. Sommerstein, Rami, et al. "Transmission of Mycobacterium chimaera from heater-cooler units during cardiac surgery despite an ultraclean air ventilation system." Emerging infectious diseases 22(6) (2016): 1008-1013.
7. Kohler, Philipp, et al. "Healthcare-associated prosthetic heart valve, aortic vascular graft, and disseminated Mycobacterium chimaera infections subsequent to open heart surgery." European heart journal 36(40) (2015): 2745-2753.

8. Achermann, Yvonne, et al. "Prosthetic valve endocarditis and bloodstream infection due to Mycobacterium chimaera." Journal of clinical microbiology 51(6) (2013): 1769-1773.

9. Infections associated with heater cooler units used in cardiopulmonary bypass and ECMO - information for healthcare providers in England. Version 1. - October 2015.

10. EU protocol for case detection, laboratory diagnosis and environmental testing of Mycobacterium chimaera infections potentially associated with heater-cooler units: case definition and environmental testing methodology - August 2015.

11. European Centre for Disease Prevention and Control. Invasive cardiovascular infection by Mycobacterium chimaera associated with 3T heater-cooler system used during openheart surgery - 18 November 2016. Stockholm: ECDC; 2016.

12. Griffith DE et coll., An official ATS/IDSA statement: diagnosis, treatment and prevention of nontuberculous mycobacterial diseases. Am J Respir Crit Care Med. 2007 Feb 15;175(4):367-416. 


\section{Annexe 1 : Liens vers les services de laboratoire provinciaux}

\begin{tabular}{|c|c|c|}
\hline Province & Liens vers les services de laboratoire provinciaux & $\begin{array}{c}\text { Personnes-ressources des } \\
\text { laboratoires }\end{array}$ \\
\hline \multirow[t]{2}{*}{ Colombie-Britannique } & $\begin{array}{l}\text { http://www.bccdc.ca/health-professionals/professional-resources/laboratory- } \\
\text { services }\end{array}$ & mel.krajden@bccdc.ca \\
\hline & & mabel.rodrigues@bccdc.ca \\
\hline \multirow[t]{2}{*}{ Alberta } & http://www.provlab.ab.ca/guide-to-services.pdf & greg.tyrrell@albertahealthservices.ca \\
\hline & & cary.shandro@albertahealthservices.ca \\
\hline \multirow[t]{2}{*}{ Saskatchewan } & http://sdcl-testviewer.ehealthsask.ca/ & paul.levett@health.gov.sk.ca \\
\hline & & dfarrell@health.gov.sk.ca \\
\hline \multirow[t]{2}{*}{ Manitoba } & http://dsmanitoba.ca/ & arendina@dsmanitoba.ca \\
\hline & & dswidinsky@dsmanitoba.ca \\
\hline \multirow[t]{2}{*}{ Ontario } & $\begin{array}{l}\text { http://www.publichealthontario.ca/en/ServicesAndTools/LaboratoryServices/ } \\
\text { Pages/Index.aspx }\end{array}$ & frances.jamieson@oahpp.ca \\
\hline & & kevin.may@oahpp.ca \\
\hline Québec & https://www.inspq.qc.ca/lspq/repertoire-des-analyses & hafid.soualhine@inspq.qc.ca \\
\hline \multirow[t]{2}{*}{ Terre-Neuve } & www.publichealthlab.ca. & jessica.kafka@easternhealth.ca \\
\hline & & robert.needle@easternhealth.ca \\
\hline \multirow[t]{2}{*}{ Nouvelle-Écosse } & $\begin{array}{l}\text { http://www.cdha.nshealth.ca/pathology-laboratory-medicine/laboratory- } \\
\text { client-support-center }\end{array}$ & david.haldane@nshealth.ca \\
\hline & & darlene.mcphee@nshealth.ca \\
\hline \multirow[t]{2}{*}{ Nouveau-Brunswick } & & hope.mackenzie@HorizonNB.ca \\
\hline & & janet.reid@HorizonNB.ca \\
\hline $\begin{array}{l}\text { Territoires du } \\
\text { Nord-Ouest }\end{array}$ & & caroline_newberry@gov.nt.ca \\
\hline Nunavut & & smarchand@gov.nu.ca \\
\hline
\end{tabular}

